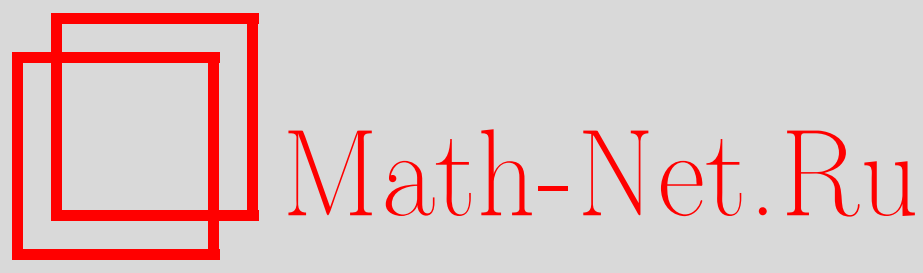

М. Бойти, Ф. Пемпинелли, А. К. Погребков, Расширенная резольвента оператора теплопроводности с солитонным потенциалом, ТМ $\Phi, 2012$, том 172, номер 2, 181-197

DOI: https://doi.org/10.4213/tmf6970

Использование Общероссийского математического портала Math-Net.Ru подразумевает, что вы прочитали и согласны с пользовательским соглашением http://www . mathnet.ru/rus/agreement

Параметры загрузки:

IP: 35.174 .16 .151

26 апреля 2023 г., 11:03:45

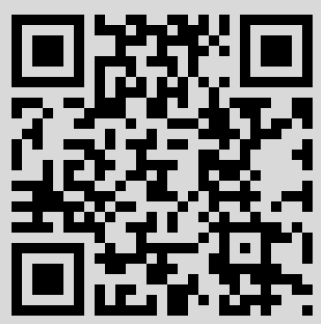


Том 172, № 2

август, 2012

(C) 2012 г. $\quad$ М. Бойти*, Ф. Пемпинелли*, А. К. Погребков ${ }^{\dagger}$

\title{
РАСШИРЕННАЯ РЕЗОЛЬВЕНТА ОПЕРАТОРА ТЕПЛОПРОВОДНОСТИ С СОЛИТОННЫМ ПОТЕНЦИАЛОМ
}

\begin{abstract}
Рассмотрен оператор теплопроводности с общим многосолитонным потенциалом, выведена его расширенная резольвента, зависящая от параметра $q \in \mathbb{R}^{2}$. Показана ее ограниченность по всем переменным и разрывность по параметру $q$. Введены функции Грина и детально исследованы их свойства.
\end{abstract}

Ключевые слова: уравнение Кадомцева-Петвиашвили, оператор теплопроводности, расширенная резольвента, солитоны.

\section{1. ВВЕДЕНИЕ}

Мы рассматриваем уравнение Кадомцева-Петвиашвили II (KП-II) [1]

$$
\left(u_{t}-6 u u_{x_{1}}+u_{x_{1} x_{1} x_{1}}\right)_{x_{1}}=-3 u_{x_{2} x_{2}}
$$

где $u=u(x, t), x=\left(x_{1}, x_{2}\right)$ и нижние индексы $x_{1}, x_{2}, t$ означают частные производные. С начала 70-х гг. известно, что это уравнение является интегрируемым [2], [3] и может считаться прототипичным $(2+1)$-мерным интегрируемым уравнением, представляя собой обобщение знаменитого уравнения Кортевега-де Фриза (КдФ). Уравнение КП-II интегрируемо, в силу того что оно ассоциировано с оператором

$$
\mathcal{L}\left(x, \partial_{x}\right)=-\partial_{x_{2}}+\partial_{x_{1}}^{2}-u(x),
$$

задающим хорошо известное уравнение теплопроводности. Спектральная теория оператора (1.1) была развита в работах [4]-[7] для вещественного потенциала $u(x)$, быстро убывающего на пространственной бесконечности. Это, однако, не является наиболее интересным случаем, поскольку уравнение КП-ІІ как раз и было предложено в работе [1] для описания двумерных слабо поперечных возмущений односолитонного решения уравнения КдФ. Основная сложность исследования таких систем связана с тем, что солитонные решения уравнений КП не убывают на пространственной бесконечности, а обладают на $x$-плоскости лучевым поведением (см., например,

${ }^{*}$ Dipartimento di Fisica, Università del Salento and Sezione INFN, Lecce, Italy

${ }^{\dagger}$ Математический институт им. В. А. Стеклова РАН, Москва, Россия.

E-mail: pogreb@mi.ras.ru 
работы [8]-[11]). Соответственно, в этом случае лишены смысла и интегральные уравнения, определяющие решения Йоста для быстроубываюшего потенциала, поскольку ядра этих уравнений не существуют.

Теория уравнения KП-II, включающая также и солитоны, все еще ждет своего построения, подобно тому как это было успешно сделано в работе [12] для уравнения КП-I. Следуя подходу указанной работы, мы обобщаем стандартный метод обратной задачи рассеяния и рассматриваем так называемое "рассеяние на нетривиальном фоне", т. е. потенциал

$$
\widetilde{u}(x)=u(x)+u^{\prime}(x),
$$

где $u(x)$ - некоторый многосолитонный потенциал, а $u^{\prime}(x)$ - гладкая, достаточно быстро убывающая по обеим своим переменным функция, которую можно считать возмущением солитонного потенциала. Решение Йоста, отвечающее оператору $\widetilde{\mathcal{L}}$ с потенциалом $\widetilde{u}$, можно определить как решение следующего интегрального уравнения:

$$
\widetilde{\Phi}(x, \mathbf{k})=\Phi(x, \mathbf{k})+\int d y \mathcal{G}(x, y, \mathbf{k}) u^{\prime}(y) \widetilde{\Phi}(y, \mathbf{k}),
$$

где $\mathbf{k} \in \mathbb{C}$ - спектральный параметр, $\Phi(x, \mathbf{k})$ - решения Йоста, отвечающее оператору $\mathcal{L}$ с многосолитонным потенциалом $u$, а $\mathcal{G}(x, y, \mathbf{k})$ - его полная функция Грина,

$$
\left(-\partial_{x_{2}}+\partial_{x_{1}}^{2}-u(x)\right) \mathcal{G}\left(x, x^{\prime}, \mathbf{k}\right)=\delta\left(x-x^{\prime}\right) .
$$

Пусть возмущение $u^{\prime}(x)$ убывает на бесконечности быстрее чем $1 /\left(x_{1}^{2}+x_{2}^{2}\right)$. Тогда если функция Грина удовлетворяет условию ограниченности, т. е. функция

$$
G\left(x, x^{\prime}, \mathbf{k}\right)=e^{i \mathbf{k}\left(x_{1}-x_{1}^{\prime}\right)+\mathbf{k}^{2}\left(x_{2}-x_{2}^{\prime}\right)} \mathcal{G}\left(x, x^{\prime}, \mathbf{k}\right)
$$

ограничена по переменным $x, x^{\prime} \in \mathbb{R}^{2}$ и $\mathbf{k} \in \mathbb{C}$ и имеет конечные пределы на бесконечности, то ядро в уравнении (1.3) хорошо определено, и фактически можно использовать стандартную технику [4]-[7], чтобы доказать существование решения $\widetilde{\Phi}(x, \mathbf{k})$. Таким образом нам удалось развить преобразование обратной задачи рассеяния для возмущения односолитонного решения [13]. Однако случай произвольного числа солитонов все еще остается открытым для исследования. В работе [14] для этого случая мы вывели полную функцию Грина, являющуюся естественным обобщением функции Грина для убывающего потенциала. Как было показано в статьях [12] и [13], для описания особенностей решений Йоста требуются также некоторые вспомогательные функции Грина. Поэтому, чтобы иметь возможность работать с оператором теплопроводности с общим многосолитонным потенциалом, мы, следуя работе [12], вводим его расширенную резольвенту (для некоторых многосолитонных решений это было сделано в работе [15]), которая является более общим объектом, чем функция Грина. А именно, мы вводим двумерный вещественный параметр $q=\left(q_{1}, q_{2}\right)$ и рассматриваем расширенный оператор Лакса

$$
\mathcal{L}\left(x, \partial_{x}+q\right)=-\partial_{x_{2}}-q_{2}+\left(\partial_{x_{1}}+q_{1}\right)^{2}-u(x) .
$$

Тогда расширенная резольвента оператора теплопроводности (1.1) определяется как обобщенная функция $M\left(x, x^{\prime} ; q\right)$ умеренного роста по всем своим шести переменным $x, x^{\prime}, q$, удовлетворяющая дифференциальному уравнению

$$
\mathcal{L}\left(x, \partial_{x}+q\right) M\left(x, x^{\prime} ; q\right)=\mathcal{L}^{\mathrm{d}}\left(x^{\prime}, \partial_{x^{\prime}}+q\right) M\left(x, x^{\prime} ; q\right)=\delta\left(x-x^{\prime}\right),
$$


где $\mathcal{L}^{\mathrm{d}}\left(x, \partial_{x}\right)$ - оператор, дуальный к $\mathcal{L}\left(x, \partial_{x}\right)$.

Расширенную резольвенту можно рассматривать как порождающий функционал для различных функций Грина оператора $\mathcal{L}(1.1)$. Действительно, введем ядро “со шляпкой"

$$
\widehat{M}\left(x, x^{\prime} ; q\right)=e^{q\left(x-x^{\prime}\right)} M\left(x, x^{\prime} ; q\right), \quad q x=q_{1} x_{1}+q_{2} x_{2},
$$

которое, конечно, не обязано быть обобщенной функцией умеренного роста. Тем не менее легко видеть, что

$$
\mathcal{L}\left(x, \partial_{x}\right) \widehat{M}\left(x, x^{\prime} ; q\right)=\mathcal{L}^{\mathrm{d}}\left(x^{\prime}, \partial_{x^{\prime}}\right) \widehat{M}\left(x, x^{\prime} ; q\right)=\delta\left(x-x^{\prime}\right) .
$$

В частности, полная функция Грина получается в результате редукции:

$$
\mathcal{G}\left(x, x^{\prime}, \mathbf{k}\right)=\left.\widehat{M}\left(x, x^{\prime} ; q\right)\right|_{\substack{q_{1}=\operatorname{Im} \mathbf{k}, q_{2}=\operatorname{Im}^{2} \mathbf{k}-\operatorname{Re}^{2} \mathbf{k}}} .
$$

Предмет настоящей статьи - построение обобщенной функции $M\left(x, x^{\prime} ; q\right)$. В paботе [16] показано, что оператор теплопроводности (1.1) с многосолитонным потенциалом $u(x)$ может иметь левые и правые аннуляторы в некотором многоугольнике на $q$-плоскости, и, следовательно, в этом многоугольнике резольвента не существует. Мы приводим явное выражение для расширенной резольвенты и доказываем, что вне некоторого специального многоугольника (такого же, как был указан в работе [16]) она существует как обобщенная функция умеренного роста и удовлетворяет уравнению (1.6). Мы показываем, что в этой области редукция (1.9) возможна для произвольного $\mathbf{k} \in \mathbb{C}$, и в результате мы получаем полную функцию Грина из работы [14]. При этом условие, что $M\left(x, x^{\prime} ; q\right)$ является обобщенной функцией умеренного роста, приводит к тому, что для функции (1.4) выполнено условие ограниченности. В соответствии с процедурой, которой мы следовали в случае нестационарного оператора Шредингера в статье [12], описание особенностей полной функции Грина на комплексной плоскости спектрального параметра требует некоторых специальных редукций расширенной резольвенты, т. е. вспомогательных функций Грина. Мы завершаем статью детальным исследованием данных особенностей.

\section{2. ОПЕРАТОР ТЕПЛОПРОВОДНОСТИ С МНОГОСОЛИТОННЫМ ПОТЕНЦИАЛОМ И РЕШЕНИЯ ЙОСТА}

Солитонные потенциалы [8]-[11], [15]-[17] характеризуются двумя натуральными числами (топологическими зарядами) $N_{a} \geqslant 1$ и $N_{b} \geqslant 1$. Пусть $\mathcal{N}=N_{a}+N_{b}$, так что $\mathcal{N} \geqslant 2$. Введем $\mathcal{N}$ вещественных параметров

$$
\kappa_{1}<\kappa_{2}<\cdots<\kappa_{\mathcal{N}}
$$

и функции

$$
K_{n}(x)=\kappa_{n} x_{1}+\kappa_{n}^{2} x_{2}, \quad n=1, \ldots, \mathcal{N} .
$$

Пусть $e^{K(x)}=\operatorname{diag}\left\{e^{K_{n}(x)}\right\}_{n=1}^{\mathcal{N}}-$ диагональная $(\mathcal{N} \times \mathcal{N})$-матрица, $\mathcal{D}-$ постоянная $\left(\mathcal{N} \times N_{b}\right)$-матрица и $\mathcal{V}$ - "неполная матрица Вандермонда", т. е. $\left(N_{b} \times \mathcal{N}\right)$-матрица вида

$$
\mathcal{V}=\left(\begin{array}{cccc}
1 & 1 & \ldots & 1 \\
\kappa_{1} & \kappa_{2} & \ldots & \kappa_{\mathcal{N}} \\
\vdots & \vdots & \ddots & \vdots \\
\kappa_{1}^{N_{b}-1} & \kappa_{2}^{N_{b}-1} & \ldots & \kappa_{\mathcal{N}}^{N_{b}-1}
\end{array}\right)
$$


Солитонный потенциал задается как

$$
u(x)=-2 \partial_{x_{1}}^{2} \ln \tau(x),
$$

где $\tau$-функцию можно представить в виде

$$
\tau(x)=\operatorname{det}\left(\mathcal{V} e^{K(x)} \mathcal{D}\right)
$$

Для решения Йоста и дуального решения Йоста (решений для оператора теплопроводности (1.1) и его дуального соответственно) имеем

$$
\begin{array}{ll}
\Phi(x, \mathbf{k})=e^{-i \mathbf{k} x_{1}-\mathbf{k}^{2} x_{2}} \chi(x, \mathbf{k}), & \chi(x, \mathbf{k})=\frac{\tau_{\Phi}(x, \mathbf{k})}{\tau(x)}, \\
\Psi(x, \mathbf{k})=e^{i \mathbf{k} x_{1}+\mathbf{k}^{2} x_{2}} \xi(x, \mathbf{k}), & \xi(x, \mathbf{k})=\frac{\tau_{\Psi}(x, \mathbf{k})}{\tau(x)},
\end{array}
$$

причем имеют место равенства (сдвиг Мивы)

$$
\tau_{\Phi}(x, \mathbf{k})=\operatorname{det}\left(\mathcal{V} e^{K(x)}(\kappa+i \mathbf{k}) \mathcal{D}\right), \quad \tau_{\Psi}(x, \mathbf{k})=\operatorname{det}\left(\mathcal{V} \frac{e^{K(x)}}{\kappa+i \mathbf{k}} \mathcal{D}\right)
$$

где $\kappa+i \mathbf{k}=\operatorname{diag}\left\{\kappa_{n}+i \mathbf{k}\right\}_{n=1}^{\mathcal{N}}$.

Для исследования свойств потенциала и решений Йоста удобно использовать представление $\tau$-функций, которое вытекает из формулы Бине-Коши для детерминанта произведения матриц:

$$
\begin{aligned}
\tau(x) & =\frac{1}{N_{b} !} \sum_{\left\{n_{i}\right\}=1}^{\mathcal{N}} \mathcal{D}\left(\left\{n_{i}\right\}\right) V\left(\left\{n_{i}\right\}\right) \prod_{l=1}^{N_{b}} e^{K_{n_{l}}(x)} \\
\chi(x, \mathbf{k}) & =\frac{1}{N_{b} ! \tau(x)} \sum_{\left\{m_{i}\right\}=1}^{\mathcal{N}} \mathcal{D}\left(\left\{m_{i}\right\}\right) V\left(\left\{m_{i}\right\}\right) \prod_{l=1}^{N_{b}}\left(\kappa_{m_{l}}+i \mathbf{k}\right) e^{K_{m_{l}}(x)} \\
\xi(x, \mathbf{k}) & =\frac{1}{N_{b} ! \tau(x)} \sum_{\left\{n_{i}\right\}=1}^{\mathcal{N}} \mathcal{D}\left(\left\{n_{i}\right\}\right) V\left(\left\{n_{i}\right\}\right) \prod_{l=1}^{N_{b}} \frac{e^{K_{n_{l}}(x)}}{\kappa_{n_{l}}+i \mathbf{k}}
\end{aligned}
$$

где использованы обозначения для максимальных миноров матриц $\mathcal{V}$ и $\mathcal{D}$,

$$
\begin{aligned}
& V\left(\left\{n_{i}\right\}\right)=\operatorname{det}\left(\begin{array}{cccc}
1 & 1 & \ldots & 1 \\
\kappa_{n_{1}} & \kappa_{n_{2}} & \ldots & \kappa_{n_{N_{b}}} \\
\vdots & \vdots & \ddots & \vdots \\
\kappa_{n_{1}}^{N_{b}-1} & \kappa_{n_{2}}^{N_{b}-1} & \ldots & \kappa_{n_{N_{b}}}^{N_{b}-1}
\end{array}\right) \equiv \prod_{1 \leqslant i<j \leqslant N_{b}}\left(\kappa_{n_{j}}-\kappa_{n_{i}}\right), \\
& \mathcal{D}\left(\left\{n_{i}\right\}\right)=\operatorname{det}\left(\begin{array}{ccc}
\mathcal{D}_{n_{1}, 1} & \ldots & \mathcal{D}_{n_{1}, N_{b}} \\
\vdots & \ddots & \vdots \\
\mathcal{D}_{n_{N_{b}}, 1} & \ldots & \mathcal{D}_{n_{N_{b}}, N_{b}}
\end{array}\right),
\end{aligned}
$$

и $\left\{m_{i}\right\}=\left\{m_{1}, \ldots, m_{N_{b}}\right\},\left\{n_{i}\right\}=\left\{n_{1}, \ldots, n_{N_{b}}\right\}$ - неупорядоченные наборы, состоящие из $N_{b}$ индексов из множества $\{1, \ldots, \mathcal{N}\}$. Напомним, что максимальные миноры матриц удовлетворяют соотношениям Плюккера: для любых подмножеств 
$\left\{m_{1}, \ldots, m_{N_{b}}\right\}$ и $\left\{n_{1}, \ldots, n_{N_{b}}\right\}$ индексов, принимающих значения от 1 до $\mathcal{N}$, и произвольного $j \in\left\{1, \ldots, N_{b}\right\}$

$$
\begin{aligned}
\mathcal{D}\left(\left\{m_{i}\right\}\right) \mathcal{D}\left(\left\{n_{i}\right\}\right)= & \sum_{s=1}^{N_{b}} \mathcal{D}\left(m_{1}, \ldots, m_{s-1}, n_{j}, m_{s+1}, \ldots, m_{N_{b}}\right) \times \\
& \times \mathcal{D}\left(n_{1}, \ldots, n_{j-1}, m_{s}, n_{j+1}, \ldots, n_{N_{b}}\right) .
\end{aligned}
$$

Заметим, что единственными зависящими от $x$ членами в $(2.4)-(2.6)$ являются экспоненты от сумм линейных функций (2.1). Таким образом, асимптотическое поведение функции $\tau(x)$ и потенциала имеет секториальную структуру на $x$-плоскости. Для описания этой структуры при $x \rightarrow \infty$ мы вводим направления (лучи) $r_{n}$ такие, что вдоль $r_{n}$ функция $x_{1}+\left(\kappa_{n}+\kappa_{n+N_{b}}\right) x_{2}$ ограничена, а $\left(\kappa_{n+N_{b}}-\kappa_{n}\right) x_{2} \rightarrow-\infty$, $n=1, \ldots, \mathcal{N}$. Здесь мы полагаем, что индексы определены по модулю $\mathcal{N}$, так что в силу $\mathcal{N}=N_{a}+N_{b}$, скажем, $n+N_{b}=n-N_{a}$ для $n>N_{a}$. В результате мы имеем $N_{a}$ лучей в направлении $x_{2} \rightarrow-\infty$ и $N_{b}$ лучей в направлении $x_{2} \rightarrow+\infty$. Сектор $\sigma_{n}$ заметается вращением луча $r_{n}$ до луча $r_{n+1}$ против часовой стрелки. Эти секторы не пересекаются и покрывают всю $x$-плоскость за исключением лучей. В работе [9] мы доказали, что при $x \rightarrow \infty$ лидирующие экспоненты в $\tau(x)$ суть $\exp \left(\sum_{l=n}^{n+N_{b}-1} K_{l}(x)\right)$, причем каждая из них является лидирующей в соответствующем секторе $\sigma_{n}$ на $x$-плоскости. Более точно, если коэффициенты $z_{n}=V\left(n, \ldots, n+N_{b}-1\right) \mathcal{D}\left(n, \ldots, n+N_{b}-1\right)$ отличны от нуля при всех $n=1, \ldots, \mathcal{N}$ (снова при условии, что индексы определены по модулю $\mathcal{N}$ ), то функция $\tau(x)$ имеет вдоль лучей и внутри секторов следующее асимптотическое поведение:

$$
\begin{array}{ll}
x \stackrel{r_{n}}{\longrightarrow} \infty: & \tau(x)=\left(z_{n}+z_{n+1} e^{K_{N_{b}+n}(x)-K_{n}(x)}+o(1)\right) \exp \left(\sum_{l=n}^{n+N_{b}-1} K_{l}(x)\right), \\
x \stackrel{\sigma_{n}}{\longrightarrow} \infty: & \tau(x)=\left(z_{n}+o(1)\right) \exp \left(\sum_{l=n}^{n+N_{b}-1} K_{l}(x)\right) .
\end{array}
$$

Регулярность потенциала $u(x)$ на $x$-плоскости эквивалентна отсутствию нулей у функции $\tau(x)$. Для этого достаточно наложить условие, что матрица $\mathcal{D}$ совершенно неотрицательна, т. е. $\mathcal{D}\left(n_{1}, \ldots, n_{N_{b}}\right) \geqslant 0$ для всех $1 \leqslant n_{1}<\cdots<n_{N_{b}} \leqslant \mathcal{N}$. С другой стороны, из (2.9) непосредственно следует, что для получения несингулярных асимптотик потенциала достаточно потребовать выполнения неравенств $z_{n}>0$. В случае совершенно неотрицательной матрицы данное условие эквивалентно [18] условию, что все максимальные миноры матрицы $D$ положительны, т. е. она - совершенно положительная матрица.

Отметим также, что функции $\chi(x, \mathbf{k})$ и $\xi(x, \mathbf{k})$ имеют ограниченные асимптотики на $x$-плоскости, поскольку зависящие от $x$ экспоненты входят в числители и знаменатели выражений $(2.5)$ и (2.6) с коэффициентами, пропорциональными $\mathcal{D}\left(\left\{n_{i}\right\}\right)$. Это означает, что лидирующее асимптотическое поведение знаменателей функций $\chi(x, \mathbf{k})$ и $\xi(x, \mathbf{k})$ на $x$-плоскости не слабее, чем поведение их числителей. Подробности см. в работах [8], [9], [16], [17], где использованы те же обозначения. 
В дальнейшем нам потребуются значения $\chi\left(x, i \kappa_{n}\right)$ функции $\chi(x, \mathbf{k})$ и вычеты $\xi_{n}(x)$ функции $\xi(x, \mathbf{k})$ в точках $\mathbf{k}=i \kappa_{n}$. С учетом $(2.3)$ и $(2.5),(2.6)$ имеем

$$
\begin{aligned}
\chi\left(x, i \kappa_{n}\right) & =\frac{(-1)^{N_{b}}}{N_{b} ! \tau(x)} \sum_{\left\{m_{i}\right\}=1}^{\mathcal{N}} \mathcal{D}\left(\left\{m_{i}\right\}\right) V\left(\left\{m_{i}\right\}, n\right) \prod_{l=1}^{N_{b}} e^{K_{m_{l}}(x)}, \\
\xi_{n}(x) & =\frac{1}{i N_{b} ! \tau(x)} \sum_{\left\{n_{i}\right\}=1}^{\mathcal{N}} \mathcal{D}\left(\left\{n_{i}\right\}\right) \sum_{j=1}^{N_{b}} \delta_{n_{j} n}(-1)^{j-1} V\left(n_{1}, \ldots, \widehat{n_{j}}, \ldots, n_{N_{b}}\right) \prod_{l=1}^{N_{b}} e^{K_{n_{l}}(x)},
\end{aligned}
$$

где $\left\{\left\{m_{i}\right\}, n\right\}=\left\{m_{1}, \ldots, m_{N_{b}}, n\right\}$, символ $\widehat{n_{j}}$ означает, что индекс $n_{j}$ пропущен, и символ Кронекера $\delta_{n_{j} n}$ в правой части формулы (2.11) обусловлен тем фактом, что вычеты слагаемых под знаком суммы отличны от нуля, только когда $n_{j}=n$ для какого-либо $j$.

Принимая во внимание свойства аналитичности функций $\chi(x, \mathbf{k})$ и $\xi(x, \mathbf{k})$ (см. формулы $(2.5),(2.6))$, их произведение можно записать через значения $\chi\left(x, i \kappa_{n}\right)$ и $\xi_{n}(x)$ следующим образом:

$$
\chi(x, \mathbf{k}) \xi\left(x^{\prime}, \mathbf{k}\right)=1+\sum_{n=1}^{\mathcal{N}} \frac{\chi\left(x, i \kappa_{n}\right) \xi_{n}\left(x^{\prime}\right)}{\mathbf{k}-i \kappa_{n}}
$$

что также будет полезно в дальнейшем. В работе [16] мы показали, что решения Йоста удовлетворяют билинейному тождеству Хироты

$$
\sum_{n=1}^{\mathcal{N}} \Phi\left(x, i \kappa_{n}\right) \Psi_{n}\left(x^{\prime}\right)=0,
$$

где по аналогии с $(2.11) \Psi_{n}(x)$ означает вычет функции $\Psi(x, \mathbf{k})$ при $\mathbf{k}=i \kappa_{n}$.

\section{3. РАСШИРЕННАЯ РЕЗОЛЬВЕНТА $M\left(X, X^{\prime} ; Q\right)$}

Докажем, что резольвента $M\left(x, x^{\prime} ; q\right)$, т. е. обобщенная функция умеренного роста по своим шести переменным, удовлетворяющая уравнению (1.6), может быть записана в виде суммы непрерывной (в некотором смысле) и дискретной частей, определения и свойства которых даны ниже:

$$
M\left(x, x^{\prime} ; q\right)=M_{\mathrm{c}}\left(x, x^{\prime} ; q\right)+M_{\mathrm{d}}\left(x, x^{\prime} ; q\right) .
$$

Мы также укажем бесконечную область на $q$-плоскости, где $M\left(x, x^{\prime} ; q\right)$ есть функция умеренного роста, и докажем, что на самом деле $M\left(x, x^{\prime} ; q\right)$ ограничена по $q$ в этой области. Для обоих членов суммы мы используем обозначения со шляпкой, введенные в (1.7):

$$
M_{\mathrm{c}}\left(x, x^{\prime} ; q\right)=e^{-q\left(x-x^{\prime}\right)} \widehat{M}_{\mathrm{c}}\left(x, x^{\prime} ; q\right), \quad M_{\mathrm{d}}\left(x, x^{\prime} ; q\right)=e^{-q\left(x-x^{\prime}\right)} \widehat{M}_{\mathrm{d}}\left(x, x^{\prime} ; q\right) .
$$

Итак, определим сначала

$$
\begin{aligned}
\widehat{M}_{\mathrm{c}}\left(x, x^{\prime} ; q\right)= & -\frac{\operatorname{sgn}\left(x_{2}-x_{2}^{\prime}\right)}{2 \pi} \int d \alpha \theta\left(\left(q_{2}+\alpha^{2}-q_{1}^{2}\right)\left(x_{2}-x_{2}^{\prime}\right)\right) \times \\
& \times \Phi\left(x, \alpha+i q_{1}\right) \Psi\left(x^{\prime}, \alpha+i q_{1}\right),
\end{aligned}
$$


где решение Йоста $\Phi(x, \mathbf{k})$ и дуальное решение Йоста $\Psi(x, \mathbf{k})$ заданы в $(2.3)$, интегрирование ведется вдоль вещественной оси, и $\theta$ - функция Хевисайда. Свойства

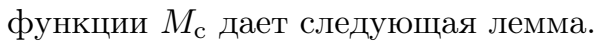

Лемма 1. Интеграл в правой части (3.3) существует, и функиия $M_{\mathrm{c}}$, заданная в (3.2), ограничена по всем переменным $x, x^{\prime}, q \in \mathbb{R}^{2}$ и имеет конечные предель на бесконечности.

ДокАЗАТЕЛьСтво. В силу (2.3) и (3.2) можно записать

$$
\begin{aligned}
M_{\mathrm{c}}\left(x, x^{\prime} ; q\right)= & M_{0}\left(x, x^{\prime} ; q\right)- \\
& -\sum_{n=1}^{\mathcal{N}} \chi\left(x, i \kappa_{n}\right) \xi_{n}\left(x^{\prime}\right) \frac{\operatorname{sgn}\left(x_{2}-x_{2}^{\prime}\right)}{2 \pi} \int d \alpha \frac{\theta\left(\left(q_{2}+\alpha^{2}-q_{1}^{2}\right)\left(x_{2}-x_{2}^{\prime}\right)\right)}{\alpha+i\left(q_{1}-\kappa_{n}\right)} \times \\
& \times e^{-i \alpha\left(x_{1}-x_{1}^{\prime}+2 q_{1}\left(x_{2}-x_{2}^{\prime}\right)\right)-\left(q_{2}+\alpha^{2}-q_{1}^{2}\right)\left(x_{2}-x_{2}^{\prime}\right)}
\end{aligned}
$$

где мы использовали формулу (2.12) для произведения $\chi(x, \mathbf{k}) \xi\left(x^{\prime}, \mathbf{k}\right)$, a $M_{0}\left(x, x^{\prime} ; q\right)$ расширенная резольвента оператора (1.5) в случае нулевого потенциала:

$$
\begin{aligned}
M_{0}\left(x, x^{\prime} ; q\right)= & -\frac{\operatorname{sgn}\left(x_{2}-x_{2}^{\prime}\right)}{2 \pi} \int d \alpha \theta\left(\left(q_{2}+\alpha^{2}-q_{1}^{2}\right)\left(x_{2}-x_{2}^{\prime}\right)\right) \times \\
& \times e^{-i \alpha\left(x_{1}-x_{1}^{\prime}+2 q_{1}\left(x_{2}-x_{2}^{\prime}\right)\right)-\left(q_{2}+\alpha^{2}-q_{1}^{2}\right)\left(x_{2}-x_{2}^{\prime}\right)} .
\end{aligned}
$$

Тогда утверждение леммы получается без труда благодаря экспоненциально убывающим множителям в (3.4) и (3.5).

Применяя оператор теплопроводности $(1.1)$ к $\widehat{M}_{\mathrm{c}}\left(x, x^{\prime} ; q\right)$ в $(3.3)$, получаем

$$
\mathcal{L}\left(x, \partial_{x}\right) \widehat{M}_{\mathrm{c}}\left(x, x^{\prime} ; q\right)=\frac{\delta\left(x_{2}-x_{2}^{\prime}\right)}{2 \pi} \int d s \Phi\left(x, s+i q_{1}\right) \Psi\left(x^{\prime}, s+i q_{1}\right) .
$$

Интеграл в правой части можно вычислить явно в силу (2.3): подставляя в него (2.8), получаем

$$
\begin{aligned}
& \frac{\delta\left(x_{2}-x_{2}^{\prime}\right)}{2 \pi} \int d s \Phi\left(x, s+i q_{1}\right) \Psi\left(x^{\prime}, s+i q_{1}\right)=\delta\left(x-x^{\prime}\right)-\delta\left(x_{2}-x_{2}^{\prime}\right) p\left(x, x^{\prime}, q_{1}\right)+ \\
& \quad+i \delta\left(x_{2}-x_{2}^{\prime}\right) \theta\left(x_{1}^{\prime}-x_{1}\right) \sum_{n=1}^{\mathcal{N}} \Phi\left(x, i \kappa_{n}\right) \Psi_{n}\left(x^{\prime}\right),
\end{aligned}
$$

где последний член исчезает вследствие (2.13). В (3.6) мы положили

$$
p\left(x, x^{\prime}, q_{1}\right)=i \sum_{n=1}^{\mathcal{N}} \theta\left(q_{1}-\kappa_{n}\right) \Phi\left(x, i \kappa_{n}\right) \Psi_{n}\left(x^{\prime}\right) .
$$

Отметим, что $p\left(x, x^{\prime}, q_{1}\right)$ не принадлежит пространству обобщенных функций Шварца, поскольку может иметь экспоненциальный рост в некоторых направлениях на $x$-плоскости [16]. Из (2.13) следует, что

$$
p\left(x, x^{\prime}, q_{1}\right)=0 \quad \text { для всех } q_{1} \notin\left[\kappa_{1}, \kappa_{\mathcal{N}}\right],
$$


и эту функцию можно переписать в любом из следующих видов:

$$
p\left(x, x^{\prime}, q_{1}\right)=\frac{i}{2} \sum_{n=1}^{\mathcal{N}} \operatorname{sgn}\left(q_{1}-\kappa_{n}\right) \Phi\left(x, i \kappa_{n}\right) \Psi_{n}\left(x^{\prime}\right) \equiv-i \sum_{n=1}^{\mathcal{N}} \theta\left(\kappa_{n}-q_{1}\right) \Phi\left(x, i \kappa_{n}\right) \Psi_{n}\left(x^{\prime}\right) .
$$

Отметим также, что по определению

$$
\mathcal{L}\left(x, \partial_{x}\right) p\left(x, x^{\prime}, q_{1}\right)=0 \quad \text { для всех } \quad q \in \mathbb{R}^{2} .
$$

Определим второе слагаемое в (3.1) как (см. также (3.2))

$$
\widehat{M}_{\mathrm{d}}\left(x, x^{\prime} ; q\right)=\mp \theta\left( \pm\left(x_{2}-x_{2}^{\prime}\right)\right) p\left(x, x^{\prime}, q_{1}\right),
$$

так что для любого выбора знака в правой части в силу (3.10) имеем

$$
\mathcal{L}\left(x, \partial_{x}\right) \widehat{M}_{\mathrm{d}}\left(x, x^{\prime} ; q\right)=\delta\left(x_{2}-x_{2}^{\prime}\right) p\left(x, x^{\prime}, q_{1}\right) .
$$

С учетом (3.6) это доказывает, что функция

$$
\widehat{M}\left(x, x^{\prime} ; q\right)=\widehat{M}_{\mathrm{c}}\left(x, x^{\prime} ; q\right)+\widehat{M}_{\mathrm{d}}\left(x, x^{\prime} ; q\right)
$$

удовлетворяет первому равенству в $(1.8)$ или функция $M\left(x, x^{\prime} ; q\right)$, заданная в $(3.1)$, в силу (1.7) удовлетворяет первому равенству в (1.6). Доказательство вторых равенств в (1.6) и (1.8) аналогично. Отметим, что вследствие (3.8)

$$
M_{\mathrm{d}}\left(x, x^{\prime} ; q\right)=0 \quad \text { для всех } q_{1} \notin\left[\kappa_{1}, \kappa_{\mathcal{N}}\right] .
$$

Таким образом, для доказательства того, что $M\left(x, x^{\prime} ; q\right)$ есть расширенная резольвента, нам осталось доказать, что $M_{\mathrm{d}}\left(x, x^{\prime} ; q\right)$ принадлежит классу обобщенных функций умеренного роста, и указать знак в правой части (3.11). Для этого нам потребуется найти в явном виде зависимость функции $M_{\mathrm{d}}\left(x, x^{\prime} ; q\right)$ от своих переменных.

Рассмотрим сначала функцию $p\left(x, x^{\prime}, q_{1}\right)$, определенную в (3.7). Подставляя равенства (2.10) и (2.11), мы в силу антисимметрии миноров матриц $\mathcal{D}$ и $\mathcal{V}$ (см. формулы (2.7)) после суммирования по $n$ получаем

$$
\begin{aligned}
p\left(x, x^{\prime}, q_{1}\right)= & \frac{-1}{N_{b} !\left(N_{b}-1\right) ! \tau(x) \tau\left(x^{\prime}\right)} \sum_{\left\{m_{i}\right\}=1}^{\mathcal{N}} \sum_{\left\{n_{i}\right\}=1}^{\mathcal{N}} \mathcal{D}\left(\left\{m_{i}\right\}\right) \mathcal{D}\left(\left\{n_{i}\right\}\right) \theta\left(q_{1}-\kappa_{n_{N_{b}}}\right) \times \\
& \times V\left(\left\{m_{i}\right\}, n_{N_{b}}\right) V\left(n_{1}, \ldots, n_{n_{N_{b}-1}}\right) \times \\
& \times \exp \left(\sum_{l=1}^{N_{b}} K_{m_{l}}(x)+K_{n_{N_{b}}}(x)+\sum_{l=1}^{N_{b}-1} K_{n_{l}}\left(x^{\prime}\right)\right) .
\end{aligned}
$$

Далее, учтем формулу (2.8) для произведения миноров матрицы $\mathcal{D}$ при $j=N_{b}$ и переобозначим индексы суммирования: $m_{s} \leftrightarrow n_{N_{b}}, s=1, \ldots, N_{b}$. Отметим, что при такой замене первый определитель Вандермонда изменяет знак, в то время как второй остается неизменным, равно как и экспонента. Итак, мы имеем

$$
p\left(x, x^{\prime}, q_{1}\right)=\frac{1}{N_{b} !\left(N_{b}-1\right) ! \tau(x) \tau\left(x^{\prime}\right)} \sum_{s=1}^{N_{b}} \sum_{\left\{m_{i}\right\}=1}^{\mathcal{N}} \sum_{\left\{n_{i}\right\}=1}^{\mathcal{N}} \mathcal{D}\left(\left\{m_{i}\right\}\right) \mathcal{D}\left(\left\{n_{i}\right\}\right) \times
$$




$$
\begin{aligned}
& \times \theta\left(q_{1}-\kappa_{m_{s}}\right) V\left(\left\{m_{i}\right\}, n_{N_{b}}\right) V\left(n_{1}, \ldots, n_{N_{b}-1}\right) \times \\
& \times \exp \left(\sum_{l=1}^{N_{b}} K_{m_{l}}(x)+K_{n_{N_{b}}}(x)+\sum_{l=1}^{N_{b}-1} K_{n_{l}}\left(x^{\prime}\right)\right) .
\end{aligned}
$$

Переобозначая теперь $m_{s}$ как $m_{N_{b}}$, мы, суммируя по $s$, получаем $N_{b}$ равных слагаемых. Умножим, наконец, (3.14) на $N_{b}$, сложим с (3.15) и разделим эту сумму на $N_{b}+1$, что дает

$$
\begin{aligned}
p\left(x, x^{\prime}, q_{1}\right)= & \frac{1}{\left(\left(N_{b}-1\right) !\right)^{2}\left(N_{b}+1\right) \tau(x) \tau\left(x^{\prime}\right)} \sum_{\left\{m_{i}\right\}=1}^{\mathcal{N}} \sum_{\left\{n_{i}\right\}=1}^{\mathcal{N}} \mathcal{D}\left(\left\{m_{i}\right\}\right) \mathcal{D}\left(\left\{n_{i}\right\}\right) \times \\
& \times\left(\theta\left(q_{1}-\kappa_{m_{N_{b}}}\right)-\theta\left(q_{1}-\kappa_{n_{N_{b}}}\right)\right) V\left(\left\{m_{i}\right\}, n_{N_{b}}\right) V\left(n_{1}, \ldots, n_{N_{b}-1}\right) \times \\
& \times \exp \left(\sum_{l=1}^{N_{b}} K_{m_{l}}(x)+K_{n_{N_{b}}}(x)+\sum_{l=1}^{N_{b}-1} K_{n_{l}}\left(x^{\prime}\right)\right),
\end{aligned}
$$

так что при всех $n=1, \ldots, \mathcal{N}-1$ для $\kappa_{n} \leqslant q_{1} \leqslant \kappa_{n+1}$

$$
\begin{aligned}
p\left(x, x^{\prime}, q_{1}\right)= & \frac{1}{\left(\left(N_{b}-1\right) !\right)^{2}\left(N_{b}+1\right) \tau(x) \tau\left(x^{\prime}\right)} \sum_{\left\{m_{i}\right\},\left\{n_{i}\right\}} \mathcal{D}\left(\left\{m_{i}\right\}\right) \mathcal{D}\left(\left\{n_{i}\right\}\right) \times \\
& \times\left(\theta\left(q_{1}-\kappa_{m_{N_{b}}}\right)-\theta\left(q_{1}-\kappa_{n_{N_{b}}}\right)\right) V\left(\left\{m_{i}\right\}, n_{N_{b}}\right) V\left(n_{1}, \ldots, n_{N_{b}-1}\right) \times \\
& \times \exp \left(\sum_{l=1}^{N_{b}} K_{m_{l}}(x)+K_{n_{N_{b}}}(x)+\sum_{l=1}^{N_{b}-1} K_{n_{l}}\left(x^{\prime}\right)\right) ;
\end{aligned}
$$

здесь и далее сумма по $\left\{m_{i}\right\},\left\{n_{i}\right\}$ подразумевает суммирование по всем наборам $m_{1}, \ldots, m_{N_{b}}$ и $n_{1}, \ldots, n_{N_{b}}$ из множества $\{1, \ldots, \mathcal{N}\}$ таким, что интервал $\left[\kappa_{n}, \kappa_{n+1}\right]$ содержится в интервале $\left[\kappa_{m_{N_{b}}}, \kappa_{n_{N_{b}}}\right]$. Отметим, что благодаря множителю $V\left(\left\{m_{i}\right\}, n_{N_{b}}\right)$ эта сумма не содержит слагаемых с $m_{N_{b}}=n_{N_{b}}$.

В силу равенств (3.2) и (3.11) мы получаем, что для всех $n=1, \ldots, \mathcal{N}-1$ при $\kappa_{n} \leqslant q_{1} \leqslant \kappa_{n+1}$

$$
\begin{aligned}
M_{\mathrm{d}}\left(x, x^{\prime} ; q\right)= & \frac{\mp \theta\left( \pm\left(x_{2}-x_{2}^{\prime}\right)\right) e^{-q\left(x-x^{\prime}\right)}}{\left(\left(N_{b}-1\right) !\right)^{2}\left(N_{b}+1\right) \tau(x) \tau\left(x^{\prime}\right)} \sum_{\left\{m_{i}\right\},\left\{n_{i}\right\}} \mathcal{D}\left(\left\{m_{i}\right\}\right) \mathcal{D}\left(\left\{n_{i}\right\}\right) \times \\
& \times\left(\theta\left(q_{1}-\kappa_{m_{N_{b}}}\right)-\theta\left(q_{1}-\kappa_{n_{N_{b}}}\right)\right) V\left(\left\{m_{i}\right\}, n_{N_{b}}\right) V\left(n_{1}, \ldots, n_{N_{b}-1}\right) \times \\
& \times \exp \left(\sum_{l=1}^{N_{b}} K_{m_{l}}(x)+K_{n_{N_{b}}}(x)+\sum_{l=1}^{N_{b}-1} K_{n_{l}}\left(x^{\prime}\right)\right)
\end{aligned}
$$

при том же условии на суммирование по $\left\{m_{i}\right\},\left\{n_{i}\right\}$. Представление (3.16) для функции $M_{\mathrm{d}}\left(x, x^{\prime} ; q\right)$ дает еще одно доказательство соотношения (3.13).

В работе [16] мы показали, что расширенный оператор (1.5) может иметь аннуляторы, когда $q$ принадлежит некоторому многоугольнику. Таким образом, оператор, обратный к (1.5) (расширенная резольвента), не может существовать при любых значениях $q$. Введем на $q$-плоскости многоугольник $\mathcal{P}$, вписанный в параболу $q_{2}=q_{1}^{2}$ 


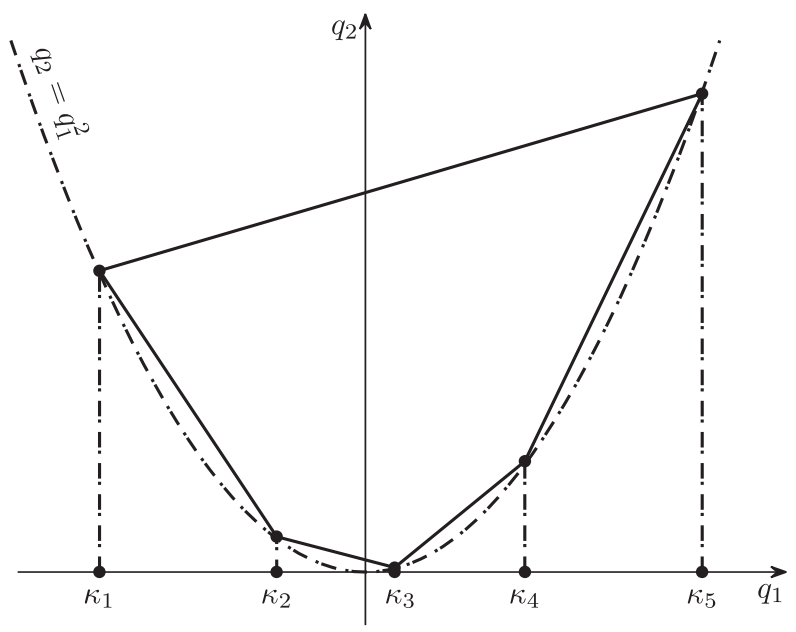

Рис. 1. Многоугольник $\mathcal{P}$ в случае $\mathcal{N}=5$.

(см. рис. 1$)$, с вершинами в точках $\left(\kappa_{n}, \kappa_{n}^{2}\right), n=1, \ldots, \mathcal{N}$, характеристическая функция которого есть

$$
\epsilon(q)=\sum_{m=1}^{\mathcal{N}-1}\left(\theta\left(q_{1}-\kappa_{n+1}\right)-\theta\left(q_{1}-\kappa_{n}\right)\right)\left(\theta\left(q_{n, n+1}\right)-\theta\left(q_{1 \mathcal{N}}\right)\right),
$$

где

$$
q_{m n}=q_{2}-\left(\kappa_{m}+\kappa_{n}\right) q_{1}+\kappa_{m} \kappa_{n} .
$$

Очевидно, что этот многоугольник делит полосу $\kappa_{1}<q_{1}<\kappa_{\mathcal{N}}$ на $q$-плоскости на две несвязные области. Более того, этот многоугольник разбивается на части, задаваемые последовательными значениями параметров $\kappa$ :

$$
\kappa_{n}<q_{1}<\kappa_{n+1}, \quad q_{n, n+1}>0, \quad n=1, \ldots, \mathcal{N}-1, \quad q_{1, \mathcal{N}}<0 .
$$

Теперь, принимая во внимание (3.13), мы можем доказать следующий результат.

ЛЕмма 2. Пусть $\tau$-функция $\left\{N_{a}, N_{b}\right\}$-солитонного потенциала $u(x)$, заданная соотношениями $(2.2),(2.4)$, удовлетворяет следующему условию: для любого подмножества $\left\{n_{1}<\cdots<n_{N_{b}}\right\} \subset\{1, \ldots, \mathcal{N}\}$ выражение

$$
\frac{1}{\tau(x)} \prod_{l=1}^{N_{b}} e^{K_{n_{l}}(x)}
$$

ограничено при всех $x$ и имеет конечные пределы на пространственной бесконечности. Тогда для всех $q$, лежащих в полосе $\kappa_{1} \leqslant q_{1} \leqslant \kappa_{\mathcal{N}}$ и вне многоугольника $\mathcal{P}$, функиия $M_{\mathrm{d}}\left(x, x^{\prime} ; q\right)$ является ограниченной при всех значениях своих аргументов, включая значения на бесконечностях, если в (3.11) выбирается верхний знак для значений $q$, лежащих в области выше многоугольника $\mathcal{P}$, и нижниц знак для значений $q$, лежащих ниже многоугольника $\mathcal{P}$. 
ДокАЗАТЕЛьство. С учетом (3.13) мы рассматриваем только значения $q$, принадлежащие полосе $\kappa_{1} \leqslant q_{1} \leqslant \kappa_{\mathcal{N}}$. Положим $z_{m n}=x_{1}+\left(\kappa_{m}+\kappa_{n}\right) x_{2}$. Тогда в силу тождества

$$
\begin{aligned}
\theta\left(q_{1}-\kappa_{m}\right) & -\theta\left(q_{1}-\kappa_{n}\right)=\operatorname{sgn}\left(z_{m n}-z_{m n}^{\prime}\right) \times \\
& \times\left[\theta\left(\left(q_{1}-\kappa_{m}\right)\left(z_{m n}-z_{m n}^{\prime}\right)\right)-\theta\left(\left(q_{1}-\kappa_{n}\right)\left(z_{m n}-z_{m n}^{\prime}\right)\right)\right]
\end{aligned}
$$

мы можем переписать (3.16) в виде

$$
\begin{aligned}
& M_{\mathrm{d}}\left(x, x^{\prime} ; q\right)=\frac{\mp \theta\left( \pm\left(x_{2}-x_{2}^{\prime}\right)\right)}{\left(\left(N_{b}-1\right) !\right)^{2}\left(N_{b}+1\right) \tau(x) \tau\left(x^{\prime}\right)} \times \\
& \times \sum_{\left\{m_{i}\right\},\left\{n_{i}\right\}} \mathcal{D}\left(\left\{m_{i}\right\}\right) \mathcal{D}\left(\left\{n_{i}\right\}\right) \operatorname{sgn}\left(z_{m_{N_{b}} n_{N_{b}}}-z_{m_{N_{b}} n_{N_{b}}}^{\prime}\right) V\left(\left\{m_{i}\right\}, n_{N_{b}}\right) V\left(n_{1}, \ldots, n_{N_{b}-1}\right) \times \\
& \times\left[\theta\left(\left(q_{1}-\kappa_{m_{N_{b}}}\right)\left(z_{m_{N_{b}} n_{N_{b}}}-z_{m_{N_{b}} n_{N_{b}}}^{\prime}\right)\right)-\theta\left(\left(q_{1}-\kappa_{n_{N_{b}}}\right)\left(z_{m_{N_{b}} n_{N_{b}}}-z_{m_{N_{b}} n_{N_{b}}}^{\prime}\right)\right)\right] \times \\
& \times \exp \left(\sum_{l=1}^{N_{b}} K_{m_{l}}(x)+K_{n_{N_{b}}}(x)+\sum_{l=1}^{N_{b}-1} K_{n_{l}}\left(x^{\prime}\right)-q\left(x-x^{\prime}\right)\right)
\end{aligned}
$$

(напомним, что суммирование по $\left\{m_{i}\right\},\left\{n_{i}\right\}$ ведется по всем наборам $m_{1}, \ldots, m_{N_{b}}$ и $n_{1}, \ldots, n_{N_{b}}$ из множества $\{1, \ldots, \mathcal{N}\}$ таким, что $\left.\left[\kappa_{n}, \kappa_{n+1}\right] \subseteq\left[\kappa_{m_{N_{b}}}, \kappa_{n_{N_{b}}}\right]\right)$.

Разобьем данное выражение на сумму двух членов в соответствии с двумя слагаемыми в квадратных скобках, подставим $q\left(x-x^{\prime}\right)$ в экспоненциальном множителе как $q\left(x-x^{\prime}\right)=K_{m}(x)-K_{m}\left(x^{\prime}\right)+q_{m n}\left(x_{2}-x_{2}^{\prime}\right)+\left(q_{1}-\kappa_{m}\right)\left(z_{m n}-z_{m n}^{\prime}\right)$, где $K_{m}(x)$ определены в (2.1), а $q_{m n}-$ в $(3.18)$, и, наконец, положим $m=m_{N_{b}}, n=n_{N_{b}}$ в первом слагаемом и $n=m_{N_{b}}, m=n_{N_{b}}$ во втором. Таким образом мы получаем

$$
M_{\mathrm{d}}\left(x, x^{\prime} ; q\right)=M^{(1)}\left(x, x^{\prime} ; q\right)+M^{(2)}\left(x, x^{\prime} ; q\right),
$$

где в каждой из полос $\kappa_{n} \leqslant q_{1} \leqslant \kappa_{n+1}, n=1, \ldots, \mathcal{N}$,

$$
\begin{aligned}
& M^{(1)}\left(x, x^{\prime} ; q\right)=\frac{\mp \theta\left( \pm\left(x_{2}-x_{2}^{\prime}\right)\right)}{\left(\left(N_{b}-1\right) !\right)^{2}\left(N_{b}+1\right)} \sum_{\left\{m_{i}\right\},\left\{n_{i}\right\}} \operatorname{sgn}\left(z_{m_{N_{b}} n_{N_{b}}}-z_{m_{N_{b}} n_{N_{b}}}^{\prime}\right) \times \\
& \times e^{-q_{m_{N_{b}}{ }^{n} N_{b}}\left(x_{2}-x_{2}^{\prime}\right)} V\left(\left\{m_{i}\right\}, n_{N_{b}}\right) V\left(n_{1}, \ldots, n_{N_{b}-1}\right) \times
\end{aligned}
$$

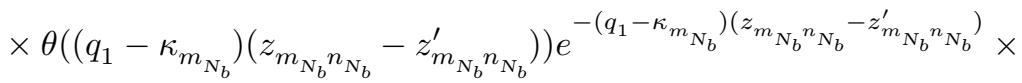

$$
\begin{aligned}
& \times \frac{\mathcal{D}\left(\left\{m_{i}\right\}\right) \exp \left(\sum_{l=1}^{N_{b}-1} K_{m_{l}}(x)+K_{n_{N_{b}}}(x)\right)}{\tau(x)} \times \\
& \times \frac{\mathcal{D}\left(\left\{n_{i}\right\}\right) \exp \left(\sum_{l=1}^{N_{b}-1} K_{n_{l}}\left(x^{\prime}\right)+K_{m_{N_{b}}}\left(x^{\prime}\right)\right)}{\tau\left(x^{\prime}\right)}
\end{aligned}
$$

И

$$
\begin{aligned}
M^{(2)}\left(x, x^{\prime} ; q\right)= & \frac{ \pm \theta\left( \pm\left(x_{2}-x_{2}^{\prime}\right)\right)}{\left(\left(N_{b}-1\right) !\right)^{2}\left(N_{b}+1\right)} \sum_{\left\{m_{i}\right\},\left\{n_{i}\right\}} \operatorname{sgn}\left(z_{m_{N_{b}} n_{N_{b}}}-z_{m_{N_{b}} n_{N_{b}}}^{\prime}\right) \times \\
& \times e^{-q_{m_{N_{b}} n_{N_{b}}}\left(x_{2}-x_{2}^{\prime}\right)} V\left(\left\{m_{i}\right\}, n_{N_{b}}\right) V\left(n_{1}, \ldots, n_{N_{b}-1}\right) \times
\end{aligned}
$$




$$
\begin{aligned}
& \times \theta\left(\left(q_{1}-\kappa_{n_{N_{b}}}\right)\left(z_{m_{N_{b}} n_{N_{b}}}-z_{m_{N_{b}} n_{N_{b}}}^{\prime}\right)\right) e^{-\left(q_{1}-\kappa_{n_{N_{b}}}\right)\left(z_{m_{N_{b}} n_{N_{b}}}-z_{m_{N_{b}}{ }^{n_{N_{b}}}}^{\prime}\right)} \times \\
& \times \frac{\mathcal{D}\left(\left\{m_{i}\right\}\right) \exp \left(\sum_{l=1}^{N_{b}} K_{m_{l}}(x)\right)}{\tau(x)} \frac{\mathcal{D}\left(\left\{n_{i}\right\}\right) \exp \left(\sum_{l=1}^{N_{b}} K_{n_{l}}\left(x^{\prime}\right)\right)}{\tau\left(x^{\prime}\right)}
\end{aligned}
$$

Правые части этих соотношений зависят от $x$ и $x^{\prime}$ посредством экспоненциальных множителей и $\tau$-функций в знаменателях. Поскольку расширенная резольвента должна принадлежать пространству обобщенных функций умеренного роста, достаточно показать, что правые части равенств (3.21) и (3.22) не могут расти на пространственной бесконечности. Рассмотрим поведение по $x$ этих двух выражений подробно.

Прежде всего, вспоминая определение (3.18) величин $q_{m n}$, отметим, что для $q$, принадлежащих $n$-й полосе $\kappa_{n} \leqslant q_{1} \leqslant \kappa_{n+1}$, таких, что $q_{n, n+1} \leqslant 0$, т. е. для значений $q$ в этой полосе, лежащих ниже многоугольника $\mathcal{P}$ или на его нижней грани, все остальные $q_{m_{N_{b}}, n_{N_{b}}}$, участвующие в суммировании, неположительны. Аналогично, если $q_{1, \mathcal{N}} \geqslant 0$, т. е. $q$ лежит в полосе $\kappa_{n} \leqslant q_{1} \leqslant \kappa_{n+1}$ над многоугольником $\mathcal{P}$ или на его верхней грани, то все остальные $q_{m_{N_{b}}, n_{N_{b}}}$, участвующие в суммировании, неотрицательны. Поэтому, если выбрать знаки в правых частях равенств (3.21) и (3.22) так, как указано в условии леммы, экспоненты во вторых строчках этих выражений убывают или ограничены, когда $x$ или $q$ стремятся к бесконечности. Экспоненты в третьих строчках в (3.21) и (3.22) убывают или по крайней мере не возрастают благодаря множителям, стоящим перед ними.

Итак, нам следует проверить поведение по $x$ и $x^{\prime}$ последних двух дробей в (3.21) и (3.22). Что касается соотношения (3.22), то в этом случае ситуация тривиальна. Две последние экспоненты в числителях этих дробей имеют те же коэффициенты (миноры матрицы $\mathcal{D})$, как в $\tau(x)$ и $\tau\left(x^{\prime}\right)$ в знаменателях, а потому эти дроби ограничены, когда $x$ и $x^{\prime}$ растут. Ситуация с (3.21) более сложная. Рассмотрим предпоследний множитель в этом выражении. Если минор $\mathcal{D}\left(\left\{m_{i}\right\}\right)$ в числителе отличен от нуля и $\mathcal{D}\left(m_{1}, \ldots, m_{N_{b}-1}, n_{N_{b}}\right) \neq 0$, то одна и та же экспонента присутствует и в числителе, и в $\tau(x)$ в знаменателе, поэтому дробь ограничена. Однако если $\mathcal{D}\left(m_{1}, \ldots, m_{N_{b}-1}, n_{N_{b}}\right)=0$, то такая экспонента не содержится в $\tau(x)$, и в направлении, где она является лидирующей (если такое направление существует), дробь растет на больших пространственных расстояниях. То же справедливо и для последнего множителя в правой части равенства (3.21). Таким образом, ограниченность этих дробей и, следовательно, всего выражения (3.21) имеет место только благодаря условию, наложенному на выражение (3.19). Лемма доказана.

ЗАмЕчаниЕ 1. Условие леммы, наложенное на выражение (3.19), является достаточным для ее справедливости, но не необходимым, и ограниченность функции $M_{\mathrm{d}}\left(x, x^{\prime} ; q\right)$ требует дополнительного исследования. Тем не менее понятно, что для совершенно положительных матриц $\mathcal{D}$ это условие справедливо. Если вместо условия совершенной положительности мы налагаем на матрицу $\mathcal{D}$ условие $z_{n}>0$, то все лидирующие экспоненты присутствуют в $\tau(x)$, как было отмечено при обсуждении соотношений (2.9). Таким образом, указанное условие леммы выполняется, если $\tau(x)$ не имеет нулей в конечной области. Чтобы избежать этих особенностей, достаточно дополнительно потребовать, чтобы матрица $\mathcal{D}$ являлась совершенно неотрицательной. 
ЗАмечание 2. Ограниченность функции $M\left(x, x^{\prime} ; q\right)$ по $q$ при $q \in \mathbb{R}^{2} \backslash \mathcal{P}$, на границах $\mathcal{P}$ и в пределах $q_{1,2} \rightarrow \infty$ следует из ограниченности функций $M_{\mathrm{c}}\left(x, x^{\prime} ; q\right)$ в $(3.4)$, а также $M^{(1)}\left(x, x^{\prime} ; q\right)$ и $M^{(2)}\left(x, x^{\prime} ; q\right)$ в $(3.21)$ и (3.22). В следующем разделе мы детально рассмотрим поведение функции $M\left(x, x^{\prime} ; q\right)$ по $q$.

ЗАмечАниЕ 3. Мы доказали, что функция $M\left(x, x^{\prime} ; q\right)$ ограничена по всем своим переменным и имеет конечное асимптотическое поведение при выполнении условий леммы 2. Это означает, что данная функция принадлежит классу обобщенных функций умеренного роста, т. е. является расширенной резольвентой оператора теплопроводности $\mathcal{L}\left(x, x^{\prime} ; q\right)$ для значений $q$, лежащих вне многоугольника $\mathcal{P}$.

\section{4. СВОЙСТВА РЕЗОЛЬВЕНТЫ И ФУНКЦИЙ ГРИНА}

4.1. Расширенная резольвента внутри и вне параболы $q_{2}=q_{1}^{2}$. Локальные свойства расширенной резольвенты легче изучать в терминах ядра (1.7). При специальной редукции (1.9) это ядро есть не что иное, как полная функция Грина $\mathcal{G}\left(x, x^{\prime}, \mathbf{k}\right)$, где $\mathbf{k} \in \mathbb{C}$ - спектральный параметр. Действительно, в этом случае разность $q_{2}-q_{1}^{2}=-\operatorname{Re}^{2} \mathbf{k}$ неположительна и $q_{1 \mathcal{N}}($ см. (3.18)) меньше или равно нулю на интервале $\kappa_{1} \leqslant q_{1} \leqslant \kappa_{\mathcal{N}}$, поэтому указанная редукция дает отображение области вне параболы $q_{2}=q_{1}^{2}$ на комплексную плоскость $\mathbf{k}$ (точнее, отображение один-в-два, поскольку редукция зависит от $|\operatorname{Re} \mathbf{k}|$, а не от $\operatorname{Re} \mathbf{k})$. Принимая во внимание условие (3.13), мы видим, что часть полосы $\kappa_{1} \leqslant q_{1} \leqslant \kappa_{\mathcal{N}}$, лежащая вне параболы, расположена ниже многоугольника $\mathcal{P}$, так что по лемме 2 следует выбрать нижний знак в (3.11). Итак, в силу (3.1), (3.3) и (3.7) получаем

$$
\begin{aligned}
\mathcal{G}\left(x, x^{\prime}, \mathbf{k}\right)= & -\frac{\operatorname{sgn}\left(x_{2}-x_{2}^{\prime}\right)}{2 \pi} \int d \alpha \theta\left(\left(\alpha^{2}-\operatorname{Re}^{2} \mathbf{k}\right)\left(x_{2}-x_{2}^{\prime}\right)\right) \times \\
& \times \Phi(x, \alpha+i \operatorname{Im} \mathbf{k}) \Psi\left(x^{\prime}, \alpha+i \operatorname{Im} \mathbf{k}\right)+ \\
& +i \theta\left(x_{2}^{\prime}-x_{2}\right) \sum_{n=1}^{\mathcal{N}} \theta\left(\operatorname{Im} \mathbf{k}-\kappa_{n}\right) \Phi\left(x, i \kappa_{n}\right) \Psi_{n}\left(x^{\prime}\right),
\end{aligned}
$$

что совпадает с функцией Грина, полученной в работе [14]. Доказанное в этой статье свойство ограниченности функции Грина (см. (1.4)) теперь следует из ограниченности резольвенты на бесконечности.

Внутренняя часть параболы, как отмечалось при обсуждении равенства (3.17), делится многоугольником $\mathcal{P}$ на область выше него $\left(q_{1, \mathcal{N}} \geqslant 0\right)$ и $\mathcal{N}-1$ линз, ограниченных параболой и ее хордами, соединяющими точки $\left(\kappa_{n}, \kappa_{n}^{2}\right)$ и $\left(\kappa_{n+1}, \kappa_{n+1}^{2}\right)$ для $n=1, \ldots, \mathcal{N}-1$ (см. рис. 1$)$. Все эти линзы находятся ниже многоугольника $\mathcal{P}$. Примем во внимание, что при $q_{2} \geqslant q_{1}^{2}$ как функция $\widehat{M}_{\mathrm{c}}\left(x, x^{\prime} ; q\right)$, так и функция $\widehat{M}_{\mathrm{d}}\left(x, x^{\prime} ; q\right)$ не зависят от $q_{2}$, и по аналогии с $(1.9)$ введем вспомогательные функции Грина

$$
\begin{aligned}
& \mathcal{G}^{+}\left(x, x^{\prime}, \operatorname{Im} \mathbf{k}\right)=\left.\widehat{M}\left(x, x^{\prime} ; q\right)\right|_{q_{1}=\operatorname{Im} \mathbf{k}}, \quad q_{2} \geqslant q_{1}^{2}, \quad q_{2} \geqslant\left(\kappa_{1}+\kappa_{\mathcal{N}}\right) q_{1}-\kappa_{1} \kappa_{\mathcal{N}}, \\
& \mathcal{G}^{-}\left(x, x^{\prime}, \operatorname{Im} \mathbf{k}\right)=\left.\widehat{M}\left(x, x^{\prime} ; q\right)\right|_{q_{1}=\operatorname{Im} \mathbf{k}}, \quad q_{2} \geqslant q_{1}^{2}, \quad q_{2} \leqslant\left(\kappa_{n}+\kappa_{n+1}\right) q_{1}-\kappa_{n} \kappa_{n+1}
\end{aligned}
$$


при $n=1, \ldots, \mathcal{N}-1$. Вновь в силу $(3.1),(3.3),(3.7)$ и (3.11) (при выборе правильного знака в соответствии с леммой 2$)$, получаем, что

$$
\begin{aligned}
\mathcal{G}^{+}\left(x, x^{\prime}, \operatorname{Im} \mathbf{k}\right)= & -\frac{\theta\left(x_{2}-x_{2}^{\prime}\right)}{2 \pi} \int d \alpha \Phi(x, \alpha+i \operatorname{Im} \mathbf{k}) \Psi\left(x^{\prime}, \alpha+i \operatorname{Im} \mathbf{k}\right)- \\
& -i \theta\left(x_{2}-x_{2}^{\prime}\right) \sum_{n=1}^{\mathcal{N}} \theta\left(\operatorname{Im} \mathbf{k}-\kappa_{n}\right) \Phi\left(x, i \kappa_{n}\right) \Psi_{n}\left(x^{\prime}\right), \\
\mathcal{G}^{-}\left(x, x^{\prime}, \operatorname{Im} \mathbf{k}\right)= & -\frac{\theta\left(x_{2}-x_{2}^{\prime}\right)}{2 \pi} \int d \alpha \Phi(x, \alpha+i \operatorname{Im} \mathbf{k}) \Psi\left(x^{\prime}, \alpha+i \operatorname{Im} \mathbf{k}\right)+ \\
& +i \theta\left(x_{2}^{\prime}-x_{2}\right) \sum_{n=1}^{\mathcal{N}} \theta\left(\operatorname{Im} \mathbf{k}-\kappa_{n}\right) \Phi\left(x, i \kappa_{n}\right) \Psi_{n}\left(x^{\prime}\right) .
\end{aligned}
$$

Ограниченность этих функций Грина следует из ограниченности расширенной резольвенты по лемме 2. А именно, благодаря (1.7) и (4.2) мы имеем, что функция $e^{\operatorname{Im} \mathbf{k}\left(x_{1}^{\prime}-x_{1}\right)+s\left(x_{2}^{\prime}-x_{2}\right)} \mathcal{G}^{+}\left(x, x^{\prime}, \operatorname{Im} \mathbf{k}\right)$ ограничена для любого вещественного $s$ такого, что

$$
s \geqslant \operatorname{Im}^{2} \mathbf{k}+\max \left\{0,\left(\kappa_{\mathcal{N}}-\operatorname{Im} \mathbf{k}\right)\left(\operatorname{Im} \mathbf{k}-\kappa_{1}\right)\right\}
$$

и функция $e^{\operatorname{Im} \mathbf{k}\left(x_{1}^{\prime}-x_{1}\right)+s\left(x_{2}^{\prime}-x_{2}\right)} \mathcal{G}^{-}\left(x, x^{\prime}, \operatorname{Im} \mathbf{k}\right)$ ограничена для любого вещественного $s$ такого, что

$$
\operatorname{Im}^{2} \mathbf{k} \leqslant s \leqslant \operatorname{Im}^{2} \mathbf{k}+\left(\kappa_{n+1}-\operatorname{Im} \mathbf{k}\right)\left(\operatorname{Im} \mathbf{k}-\kappa_{n}\right), \quad n=1, \ldots, \mathcal{N}-1 .
$$

4.2. Особенности функций Грина. Для описания особенностей рассматриваемых редукций резольвенты удобно использовать для нее представление (3.4), так что в силу $(1.7),(2.3),(3.12)$ и $(3.7),(3.11)$ можно записать

$$
\begin{aligned}
& \widehat{M}\left(x, x^{\prime} ; q\right)=\widehat{M}_{0}\left(x, x^{\prime} ; q\right)- \\
& \quad-\sum_{n=1}^{\mathcal{N}} \Phi\left(x, i \kappa_{n}\right) \Psi_{n}\left(x^{\prime}\right)\left\{\frac{\operatorname{sgn}\left(x_{2}-x_{2}^{\prime}\right)}{2 \pi} \int d \alpha \frac{\theta\left(\left(q_{2}+\alpha^{2}-q_{1}^{2}\right)\left(x_{2}-x_{2}^{\prime}\right)\right)}{\alpha+i\left(q_{1}-\kappa_{n}\right)} \times\right. \\
& \left.\quad \times e^{\left(q_{1}-\kappa_{n}-i \alpha\right)\left(x_{1}-x_{1}^{\prime}\right)-\left(\left(\alpha+i q_{1}\right)^{2}+\kappa_{n}^{2}\right)\left(x_{2}-x_{2}^{\prime}\right)} \pm i \theta\left( \pm\left(x_{2}-x_{2}^{\prime}\right)\right) \theta\left(q_{1}-\kappa_{n}\right)\right\},
\end{aligned}
$$

где вследствие (1.7) и (3.5)

$$
\begin{aligned}
\widehat{M}_{0}\left(x, x^{\prime} ; q\right)= & -\frac{\operatorname{sgn}\left(x_{2}-x_{2}^{\prime}\right)}{2 \pi} \int d \alpha \theta\left(\left(q_{2}+\alpha^{2}-q_{1}^{2}\right)\left(x_{2}-x_{2}^{\prime}\right)\right) \times \\
& \times e^{\left(q_{1}-i \alpha\right)\left(x_{1}-x_{1}^{\prime}\right)+\left(q_{1}-i \alpha\right)^{2}\left(x_{2}-x_{2}^{\prime}\right)},
\end{aligned}
$$

и знак в правой части (4.5) должен быть выбран в соответствии с леммой 2. Легко видеть, что вне параболы $q_{2}=q_{1}^{2}$ ядро $\widehat{M}\left(x, x^{\prime} ; q\right)$ - непрерывная функция по переменной $q$ при $q \neq\left(\kappa_{n}, \kappa_{n}^{2}\right), n=1, \ldots, \mathcal{N}$.

Из (4.5), используя редукцию (1.9), мы получаем для полной функции Грина (4.1) следующее представление:

$$
\mathcal{G}\left(x, x^{\prime}, \mathbf{k}\right)=-\frac{\operatorname{sgn}\left(x_{2}-x_{2}^{\prime}\right)}{2 \pi} \int_{\operatorname{Im} \mathbf{k}^{\prime}=\operatorname{Im} \mathbf{k}} d \operatorname{Re} \mathbf{k}^{\prime} \theta\left(\left(\operatorname{Re}^{2} \mathbf{k}^{\prime}-\operatorname{Re}^{2} \mathbf{k}\right)\left(x_{2}-x_{2}^{\prime}\right)\right) \times
$$




$$
\begin{aligned}
& \times e^{-i \mathbf{k}^{\prime}\left(x_{1}-x_{1}^{\prime}\right)-\mathbf{k}^{\prime 2}\left(x_{2}-x_{2}^{\prime}\right)}-\sum_{n=1}^{\mathcal{N}} \Phi\left(x, i \kappa_{n}\right) \Psi_{n}\left(x^{\prime}\right) \times \\
& \times\left\{\frac{\operatorname{sgn}\left(x_{2}-x_{2}^{\prime}\right)}{2 \pi} \int_{\operatorname{Im} \mathbf{k}^{\prime}=\operatorname{Im} \mathbf{k}} d \operatorname{Re} \mathbf{k}^{\prime} \frac{\theta\left(\left(\operatorname{Re}^{2} \mathbf{k}^{\prime}-\operatorname{Re}^{2} \mathbf{k}\right)\left(x_{2}-x_{2}^{\prime}\right)\right)}{\mathbf{k}^{\prime}-i \kappa_{n}} \times\right. \\
& \left.\times e^{-\left(\kappa_{n}+i \mathbf{k}^{\prime}\right)\left(x_{1}-x_{1}^{\prime}\right)-\left(\kappa_{n}^{2}+\mathbf{k}^{\prime 2}\right)\left(x_{2}-x_{2}^{\prime}\right)}-\frac{i}{2} \theta\left(x_{2}^{\prime}-x_{2}\right) \operatorname{sgn}\left(\operatorname{Im} \mathbf{k}-\kappa_{n}\right)\right\},
\end{aligned}
$$

где для $p\left(x, x^{\prime}, q_{1}\right)$ мы воспользовались выражением (3.9). В силу предыдущего обсуждения формула (4.6) определяет непрерывную функцию переменной $\mathbf{k} \in \mathbb{C}$ при всех $\mathbf{k} \neq i \kappa_{n}$.

Для изучения поведения функции Грина вблизи точек $\mathbf{k}=i \kappa_{n}$ рассмотрим сначала функцию Грина $\mathcal{G}^{+}(4.3)$. В силу соотношения (4.5) мы имеем

$$
\begin{aligned}
& \mathcal{G}^{+}\left(x, x^{\prime}, \operatorname{Im} \mathbf{k}\right)=-\frac{\theta\left(x_{2}-x_{2}^{\prime}\right)}{2 \pi}\left\{\int_{\operatorname{Im} \mathbf{k}^{\prime}=\operatorname{Im} \mathbf{k}} d \operatorname{Re} \mathbf{k}^{\prime} e^{-i \mathbf{k}^{\prime}\left(x_{1}-x_{1}^{\prime}\right)-\mathbf{k}^{\prime 2}\left(x_{2}-x_{2}^{\prime}\right)}+\right. \\
& \quad+\sum_{n=1}^{\mathcal{N}} \Phi\left(x, i \kappa_{n}\right) \Psi_{n}\left(x^{\prime}\right)\left(\int_{\operatorname{Im} \mathbf{k}^{\prime}=\operatorname{Im} \mathbf{k}} d \operatorname{Re} \mathbf{k}^{\prime} \frac{e^{-\left(\kappa_{n}+i \mathbf{k}^{\prime}\right)\left(x_{1}-x_{1}^{\prime}\right)-\left(\kappa_{n}^{2}+\mathbf{k}^{\prime 2}\right)\left(x_{2}-x_{2}^{\prime}\right)}}{\mathbf{k}^{\prime}-i \kappa_{n}}+\right. \\
& \left.\left.\quad+i \pi \operatorname{sgn}\left(\operatorname{Im} \mathbf{k}-\kappa_{n}\right)\right)\right\}
\end{aligned}
$$

Легко видеть, что эта функция Грина непрерывна по $\operatorname{Im} \mathbf{k}$ и, более того, функция $\mathcal{G}^{+}\left(x, x^{\prime}, \operatorname{Im} \mathbf{k}\right)$ не зависит от $\operatorname{Im} \mathbf{k}$. Действительно, дифференцируя по $\operatorname{Im} \mathbf{k}$, мы используем, что экспоненты аналитичны по переменной $\mathbf{k}^{\prime}=\operatorname{Re} \mathbf{k}^{\prime}+i \operatorname{Im} \mathbf{k}$ и быстро убывают при $\operatorname{Re} \mathbf{k}^{\prime} \rightarrow \infty$, а также, что $\bar{\partial}_{\mathbf{k}^{\prime}}\left(\mathbf{k}^{\prime}-i \kappa_{n}\right)^{-1}=\pi \delta\left(\operatorname{Re} \mathbf{k}^{\prime}\right) \delta\left(\operatorname{Im} \mathbf{k}^{\prime}-\kappa_{n}\right)$. Таким образом, производные первого слагаемого и каждого слагаемого под знаком суммы независимо равны нулю, так что можно положить

$$
\begin{aligned}
\mathcal{G}^{+}\left(x, x^{\prime}\right)= & -\frac{\theta\left(x_{2}-x_{2}^{\prime}\right)}{2 \pi}\left\{\int d \alpha e^{-i \alpha\left(x_{1}-x_{1}^{\prime}\right)-\alpha^{2}\left(x_{2}-x_{2}^{\prime}\right)}+\right. \\
& \left.+\sum_{n=1}^{\mathcal{N}} \Phi\left(x, i \kappa_{n}\right) \Psi_{n}\left(x^{\prime}\right) \int \frac{d \alpha}{\alpha} e^{-i \alpha\left(x_{1}-x_{1}^{\prime}+2 \kappa_{n}\left(x_{2}-x_{2}^{\prime}\right)\right)-\alpha^{2}\left(x_{2}-x_{2}^{\prime}\right)}\right\},
\end{aligned}
$$

где интегралы понимаются в смысле главного значения. Результат также может быть вычислен явно в терминах гипергеометрических функций.

С другой стороны, используя (4.3) и (4.4), мы непосредственно имеем, что

$$
\mathcal{G}^{-}\left(x, x^{\prime}, \operatorname{Im} \mathbf{k}\right)=\mathcal{G}^{+}\left(x, x^{\prime}\right)+\frac{i}{2} \sum_{n=1}^{\mathcal{N}} \operatorname{sgn}\left(\operatorname{Im} \mathbf{k}-\kappa_{n}\right) \Phi\left(x, i \kappa_{n}\right) \Psi_{n}\left(x^{\prime}\right),
$$

и функция $\mathcal{G}^{-}\left(x, x^{\prime}, \operatorname{Im} \mathbf{k}\right)$ разрывна при всех $\operatorname{Im} \mathbf{k}=\kappa_{n}$, точнее,

$$
\mathcal{G}^{-}\left(x, x^{\prime}, \kappa_{n}+0\right)-\mathcal{G}^{-}\left(x, x^{\prime}, \kappa_{n}-0\right)=i \Phi\left(x, i \kappa_{n}\right) \Psi_{n}\left(x^{\prime}\right), \quad n=2, \ldots, \mathcal{N}-1 .
$$

Таким образом, рассматривая разность выражений, данных в (4.6) и (4.7), мы выводим, что полная функция Грина может быть представлена в виде

$$
\mathcal{G}\left(x, x^{\prime}, \mathbf{k}\right)=\mathcal{G}_{\mathrm{reg}}\left(x, x^{\prime}, \mathbf{k}\right)+\mathcal{G}_{\Delta}\left(x, x^{\prime}, \mathbf{k}\right),
$$


где

$$
\begin{aligned}
\mathcal{G}_{\mathrm{reg}}\left(x, x^{\prime}, \mathbf{k}\right)= & \mathcal{G}^{+}\left(x, x^{\prime}\right)+\frac{1}{2 \pi} \int_{\substack{\left|\operatorname{Re} \mathbf{k}^{\prime}\right| \leqslant|\operatorname{Re} \mathbf{k}|, \operatorname{Im} \mathbf{k}^{\prime}=\operatorname{Im} \mathbf{k}}} d \operatorname{Re} \mathbf{k}^{\prime} e^{-i \mathbf{k}^{\prime}\left(x_{1}-x_{1}^{\prime}\right)-\mathbf{k}^{\prime 2}\left(x_{2}-x_{2}^{\prime}\right)}+ \\
& +\frac{1}{2 \pi} \sum_{n=1}^{\mathcal{N}} \Phi\left(x, i \kappa_{n}\right) \Psi_{n}\left(x^{\prime}\right) \times \\
& \times \int_{\left|\operatorname{Re} \mathbf{k}^{\prime}\right| \leqslant|\operatorname{Re} \mathbf{k}|,} d \operatorname{Re} \mathbf{k}^{\prime} \frac{e^{-\left(\kappa_{n}+i \mathbf{k}^{\prime}\right)\left(x_{1}-x_{1}^{\prime}\right)-\left(\kappa_{n}^{2}+\mathbf{k}^{\prime 2}\right)\left(x_{2}-x_{2}^{\prime}\right)}-1}{\mathbf{k}^{\prime}-i \kappa_{n}} .
\end{aligned}
$$

Последний интеграл был регуляризован вычитанием единицы, что компенсирует ноль в знаменателе при $\mathbf{k}^{\prime}=i \kappa_{n}$. Соответственно,

$$
\mathcal{G}_{\Delta}\left(x, x^{\prime}, \mathbf{k}\right)=\frac{1}{i \pi} \sum_{n=1}^{\mathcal{N}} \Phi\left(x, i \kappa_{n}\right) \Psi_{n}\left(x^{\prime}\right) \operatorname{arcctg} \frac{\operatorname{Im} \mathbf{k}-\kappa_{n}}{|\operatorname{Re} \mathbf{k}|}
$$

где член, пропорциональный левой части в (2.13), был опущен. Это выражение, очевидно, разрывно при $\mathbf{k}=i \kappa_{n}, n=1, \ldots, \mathcal{N}$, и только в этих точках. А именно,

$$
\begin{aligned}
\mathcal{G}_{\Delta}\left(x, x^{\prime}, \mathbf{k}\right)= & \frac{1}{i \pi} \Phi\left(x, i \kappa_{n}\right) \Psi_{n}\left(x^{\prime}\right) \operatorname{arcctg} \frac{\operatorname{Im} \mathbf{k}-\kappa_{n}}{|\operatorname{Re} \mathbf{k}|}- \\
& -i \sum_{m=n+1}^{\mathcal{N}} \Phi\left(x, i \kappa_{m}\right) \Psi_{m}\left(x^{\prime}\right)+o(1), \quad \mathbf{k} \sim i \kappa_{n}, \quad n=1, \ldots, \mathcal{N} .
\end{aligned}
$$

Отметим также, что в силу (4.11)-(4.13)

$$
\left.\mathcal{G}\left(x, x^{\prime}, \mathbf{k}\right)\right|_{\operatorname{Re} \mathbf{k}=0}=\mathcal{G}^{+}\left(x, x^{\prime}\right)-\frac{i}{2} \sum_{n=1}^{\mathcal{N}} \operatorname{sgn}\left(\kappa_{n}-\operatorname{Im} \mathbf{k}\right) \Phi\left(x, i \kappa_{n}\right) \Psi_{n}\left(x^{\prime}\right),
$$

где мы считаем, что $\operatorname{Im} \mathbf{k} \neq \kappa_{n}, n=1, \ldots, \mathcal{N}$. Вследствие (2.13) и (4.9) это дает

$$
\left.\mathcal{G}\left(x, x^{\prime}, \mathbf{k}\right)\right|_{\operatorname{Re} \mathbf{k}=0}=\mathcal{G}^{-}\left(x, x^{\prime}, \operatorname{Im} \mathbf{k}\right) .
$$

С другой стороны, в окрестностях точек $i \kappa_{n}$ мы имеем

$$
\begin{aligned}
\mathcal{G}\left(x, x^{\prime}, \mathbf{k}\right)= & \mathcal{G}^{+}\left(x, x^{\prime}\right)+\frac{1}{i \pi} \Phi\left(x, i \kappa_{n}\right) \Psi_{n}\left(x^{\prime}\right) \operatorname{arcctg} \frac{\operatorname{Im} \mathbf{k}-\kappa_{n}}{|\operatorname{Re} \mathbf{k}|}- \\
& -i \sum_{m=n+1}^{\mathcal{N}} \Phi\left(x, i \kappa_{m}\right) \Psi_{m}\left(x^{\prime}\right)+o(1), \quad \mathbf{k} \sim i \kappa_{n}, \quad n=1, \ldots, \mathcal{N} .
\end{aligned}
$$

В последующей публикации мы покажем, что эти свойства функций Грина позволяют решить проблему, сформулированную во введении, т. е. обобщить метод обратной задачи рассеяния на случай возмущенных многосолитонных потенциалов (1.2) оператора теплопроводности (1.1).

Благодарности. Работа частично поддержана РФФИ (грант № 11-01-00440), Программой поддержки ведущих научных школ (грант НШ-4612.2012.1), Программой PAH "Математические методы нелинейной динамики", INFN, MIUR (грант PRIN 2008 "Geometrical methods in the theory of nonlinear integrable systems") и Консорциумом E.I.N.S.T.E.I.N. 


\section{Список литературы}

[1] Б. Б. Кадомцев, В. И. Петвиашвили, Докл. АН СССР, 192 (1970), 753-756.

[2] В. С. Дрюма, Писъма в ЖЭТФ, 19:12 (1974), 753-755.

[3] В. Е. Захаров, А. Б. Шабат, Функи. анализ и его прил., 8:3 (1974), 43-53.

[4] M. J. Ablowitz, D. Bar Yaacov, A. S. Fokas, Stud. Appl. Math., 69:2 (1983), 135-143.

[5] В. Д. Липовский, Функи. анализ и его прил., 20:4 (1986), 35-45.

[6] M. V. Wickerhauser, Commun. Math. Phys., 108:1 (1987), 67-89.

[7] П. Г. Гриневич, С. П. Новиков, Функи. анализ и его прил., 22:1 (1988), 23-33.

[8] M. Boiti, F. Pempinelli, A. Pogrebkov, B. Prinari, Inverse Problems, 17:4 (2001), 937-957.

[9] М. Бойти, Ф. Пемпинелли, А. К. Погребков, ТМФ, 168:1 (2011), 13-23.

[10] S. Chakravarty, Y. Kodama, Stud. Appl. Math., 123:1 (2009), 83-151.

[11] Y. Kodama, J. Phys. A, 43:43 (2010), 434004, 54 pp., arXiv: 1004.4607.

[12] M. Boiti, F. Pempinelli, A. K. Pogrebkov, J. Math. Phys., 47:12 (2006), 123510, 43 pp.

[13] M. Boiti, F. Pempinelli, A. K. Pogrebkov, B. Prinari, J. Math. Phys., 43:2 (2002), 1044-1062.

[14] M. Boiti, F. Pempinelli, A. K. Pogrebkov, Green's function of heat operator with pure soliton potential, arXiv: 1201.0152.

[15] М. Бойти, Ф. Пемпинелли, А. К. Погребков, Б. Принари, ТМФ, 159:3 (2009), 364-378, arXiv: 0901.3857.

[16] M. Boiti, F. Pempinelli, A. Pogrebkov, J. Math Phys., 52:8 (2011), 083506, 22 pp., arXiv: 1105.3681.

[17] М. Бойти, Ф. Пемпинелли, А. К. Погребков, Б. Принари, ТМФ, 165:1 (2010), 3-24, arXiv: 0911.1675.

[18] М. Гехтман, М. Шапиро, частное сообщение, 2012.

Поступила в редакцию 13.03.2012 